\title{
Brandon N. Owens, The Wind Power Story: A Century of Innovation That Reshaped the Global Energy Landscape
}

Hoboken, John Wiley \& Sons Inc/IEEE Press, 2019

Philippe Bruyerre

\section{OpenEdition}

\section{Journals}

Édition électronique

URL : http://journals.openedition.org/artefact/7157

DOI : 10.4000/artefact.7157

ISSN : 2606-9245

Éditeur :

Association Artefact. Techniques histoire et sciences humaines, Presses universitaires du Midi

Édition imprimée

Date de publication : 7 janvier 2021

Pagination : 477-480

ISBN : 978-2-8107-0706-5

ISSN : 2273-0753

\section{Référence électronique}

Philippe Bruyerre, «Brandon N. Owens, The Wind Power Story: A Century of Innovation That Reshaped the Global Energy Landscape », Artefact [En ligne], 13 | 2020, mis en ligne le 23 décembre 2020, consulté le 25 décembre 2020. URL : http://journals.openedition.org/artefact/7157 ; DOI : https://doi.org/ 10.4000/artefact.7157

Ce document a été généré automatiquement le 25 décembre 2020.

\section{cc)}

Artefact, Techniques, histoire et sciences humaines est mise à disposition selon les termes de la Licence Creative Commons Attribution - Pas d'Utilisation Commerciale - Pas de Modification 4.0 International. 


\title{
Brandon N. Owens, The Wind Power Story: A Century of Innovation That Reshaped the Global Energy Landscape
}

Hoboken, John Wiley \& Sons Inc/IEEE Press, 2019

\author{
Philippe Bruyerre
}

\section{RÉFÉRENCE}

Brandon N. Owens, The Wind Power Story: A Century of Innovation That Reshaped the Global Energy Landscape, Hoboken, John Wiley \& Sons Inc/IEEE Press, 2019, 368 p.

1 Ce livre propose au lecteur un voyage à travers l'histoire de l'énergie éolienne de ses origines (1888) à nos jours (2018). Il s'agit en fait d'une histoire de l'énergie éolienne électrique, l'éolienne (wind turbine) résultant selon l'auteur d'une fusion entre le moulin à vent (windmill) et l'électricité. L'ambition du livre, annoncée par son sous-titre, est de raconter cent trente années d'innovation qui ont remodelé le "paysage énergétique global ».

2 Le livre comprend vingt chapitres, eux-mêmes constitués de trois à six sous-chapitres, rendant la lecture dynamique, agrémentée de plus de vingt illustrations d'éoliennes dessinées au trait. Les notes et la bibliographie sont regroupées à la fin de chaque chapitre (ce qui ne facilite pas la recherche d'une référence), un index complète enfin l'ouvrage. Sa grande originalité est de proposer une histoire globale dont les acteurs sont américains, européens (allemands, danois, espagnols et français) et asiatiques (chinois et indien). L'innovation dans l'éolien est nationale, mais qu'en est-il dans un contexte "global»? L'ouvrage vient combler un vide car l'histoire de l'éolien électrique est souvent diluée dans des histoires des énergies dites "nouvelles", « renouvelables » ou "alternatives », les polémiques contemporaines ayant largement contribué à cette mise à l'écart. 
3 L'auteur, économiste de l'énergie, est employé de General Electric, une entreprise qui se définit comme "globale ». Il propose une histoire articulée autour de sept grandes phases suivant un schéma évolutionniste. Cette structuration n'est cependant pas explicite dans l'ouvrage - elle figure seulement dans des documents publiés en ligne avant la publication - ce que l'on peut regretter car cette structure, même si elle est fragile, aurait permis au lecteur de mieux comprendre l'approche "globale» de l'auteur.

4 L'ouvrage commence par la phase des « pionniers » (1887-années 1920) marquée par les premières tentatives de Brush (États-Unis), La Cour (Danemark) et des petites éoliennes de Jacobs (États-Unis), elle est suivie par celle des « constructeurs » avec les premières grandes éoliennes russe (1931) et américaine (1941), puis les essais danois et allemands (1957-1958). Suite à la «crise de l'énergie» des années 1970 et 1980, les " administrateurs » prennent les commandes avec les éoliennes de la NASA (États-Unis) et Growian (Allemagne). Leurs résultats mitigés laissent la place aux « fondateurs » des années 1980 avec des entreprises comme US Windpower (États-Unis), Vestas (Danemark) et Enercon (Allemagne). Les « innovateurs radicaux » (disruptors) prennent la relève dans les années 1990 et développent les éoliennes modernes avec les machines E40 (Enercon), V33 (US Windpower) et V80 (Vestas). Le marché est ensuite dominé par les "globalisateurs» (1994-2000) avec les sociétés actives sur le marché mondial comme Gamesa (Espagne), Suzlon (Inde) et Goldwind (Chine). La dernière phase - que l'auteur nomme la «frontière finale »- est celle des "explorateurs", elle est caractérisée par l'éolien offshore qui fait l'objet du dernier chapitre avec la centrale offshore Vindeby (Danemark) et les machines de très grande puissance de SiemensGamesa et General Electric Haliade X (220 m de diamètre, $12 \mathrm{MW}$ ).

5 Comme souvent, chercher à regrouper des initiatives différentes insérées dans des contextes très différents est un exercice difficile. L'industriel Brush et le professeur La Cour sont certes tous deux des "pionniers", mais le premier installe une seule machine alors que le second développe de nombreuses centrales éoliennes rurales au Danemark. Intégrer dans une même phase - celle des «administrateurs » - des projets aussi éloignés que ceux de la NASA (impliquant de très grandes entreprises) et de Tvind (éolienne auto-construite par une école technique) semble bien téméraire. Cette mise en « phases » d'une histoire plurielle n'éclaire pas toujours le lecteur.

6 L'auteur fait une large place aux pays autres que les États-Unis, ce qui est louable. Dans certains cas, le tropisme américain ressurgit comme avec Kenetech placé à côté d'Enercon et de Vestas ou encore General Electric, mais cela ne gêne pas la lecture. On soulignera les chapitres dédiés à l'Inde et la Chine, une rareté. Si les sources sont diversifiées, elles sont parfois très limitées. Pour l'Europe, Owens s'appuie sur des sources secondaires, en particulier sur un ouvrage danois ${ }^{1}$ dont les auteurs fondaient déjà leurs propos sur la littérature secondaire, notamment dans le chapitre "The French Connection » (un titre un peu déplacé). Il évoque une "stratégie d'EDF » dans l'éolien dès 1947, l'échec de Romani n'est pas analysé et les pales en fibre de verre de Neyrpic (1959) ne sont pas mentionnées. L'auteur reprend ici le grand récit éolien danois : La Cour d'abord (1896), Juul son élève ensuite (1957) et par fusion avec les pales en composite de l'Allemand Hütter, l'éolienne danoise (1980), l'éolienne moderne. Dans ce schéma, la place de l'Allemand Tacke Windtechnik, acheté par l'entreprise américaine Zond, puis General Electric, est logiquement minimisée et le fabricant 
RePower oublié, alors que la machine allemande de 1,5 MW de 1995 constitue une « rupture » par rapport à l'éolienne danoise des années 1980.

7 Les notions de base relatives aux aspects techniques sont présentées dans l'ouvrage, même si certaines sont mal expliquées. Par exemple, le rated power (puissance nominale) est présenté comme la puissance correspondant au rendement optimal, alors qu'il s'agit de la puissance de la génératrice installée dans l'éolienne. Il est encore plus intrigant d'utiliser le contenu d'un brevet d'invention, certes américain - celui de Stuart et Fales datant de 1926 - pour expliquer que la puissance d'une éolienne augmente avec sa vitesse de rotation pour une vitesse de vent donnée. Une mauvaise lecture d'un graphique, au demeurant peu pertinent, lui fait écrire que le passage d'un rapport d'avance de 2 (éoliennes multipales) à 12 (éolienne rapide à hélice) accroît la puissance de l'éolienne de moins de $10 \mathrm{~kW}$ à plus de $60 \mathrm{~kW}$ (p.24)! Deux pages plus loin, l'auteur donne $15 \%$ de coefficient de performance pour l'éolienne multipale de Brush et $40 \%$ pour celle de Stuart et Fales (une valeur très exagérée), soit un rapport de puissance inférieur à trois (plutôt deux en fait). De plus, aucune présentation de ce qu'est la production d'énergie d'une éolienne n'est introduite, ce qui est déroutant pour un ouvrage qui se présente comme une histoire de l'énergie.

8 Par ailleurs, le contexte social est pratiquement absent : les lecteurs ne trouveront dans ce volume aucune indication sur les impacts sur l'environnement (paysage, bruit...), sur les réseaux électriques (intermittence, priorité d'achat...) ou sur la société (l'éolien comme solution au changement climatique), simplement une longue marche vers des machines encore plus puissantes, toujours plus gigantesques.

Cet ouvrage est celui d'un économiste de l'énergie et doit être pris comme tel. Il apporte certes d'utiles références sur les acteurs, même s'il est parfois difficile de les resituer dans leur contexte national et global sans simplifier les dynamiques à l'œuvre, qu'il s'agisse des difficultés techniques ou de l'acceptation sociale. L'ouvrage montre aussi l'intérêt d'une histoire sectorielle de l'énergie: les dynamiques techniques et sociales qui façonnent le développement d'une source d'énergie sont propres au secteur d'activité, dominé dans le cas de l'éolien par les matériaux (pales composites) et l'électromécanique (vitesse variable). Placer l'énergie éolienne sous la bannière des « énergies nouvelles-renouvelables-alternatives-non fossiles... » ne permet pas toujours de comprendre ce que font les acteurs dans un tel contexte.

10 À propos de cet ouvrage "globalement » (le mot ne nous quitte pas après sa lecture) passionnant, on peut formuler une dernière remarque sur son positionnement éditorial : pour un ouvrage qui ne se prétend pas académique, son prix élevé ( 75 \$ / $67 €)$ le rend difficile d'accès à l'honnête homme qui recherche une information sur cette étrange « source d'énergie » qu'est l'éolien. 


\section{BIBLIOGRAPHIE}

MAEGAARD Preben, KRENZ Anna et PALZ Wolfgang (dir.), Wind Power for the World-International Reviews and Developments, Boca Raton, Taylor \& Francis Group (coll. «Pan Stanford Series on Renewable Energy »), 2013.

\section{NOTES}

1. Maegaard, Krenz et Palz (dir.), 2013. 